\title{
Biopsychosocial Intervention for Stroke Carers (BISC): A Feasibility Randomised Controlled Trial
}

\author{
M F Walker ${ }^{\text {*** }}$, L Condon ${ }^{1}$, P Benford ${ }^{1}$, R Fisher ${ }^{1}$, O Matias ${ }^{2}$, S Birchall ${ }^{1}$, C Cobley ${ }^{3}$, C Greensmith ${ }^{4}$,
}

E Kontou ${ }^{1}$, N Sprigg ${ }^{2}$, S Thomas ${ }^{1}$, M R Golding-Day ${ }^{1}$ J Fletcher-Smith ${ }^{1}$, \& P J Whitehead ${ }^{1}$

\section{BACKGROUND \& AIMS}

Enhancing the wellbeing of carers is a national priority, yet despite this, few services or interventions have been developed, evaluated or implemented specifically for carers. Carers of stroke survivors may experience anxiety, frustration, sleep disturbance, stress and/or depression. ${ }^{1-4}$ Such deterioration in the health of the caregiver can further impact upon the stroke survivor's health and quality of life. However, attention is typically directed towards the stroke survivor's recovery and rehabilitation and, as such, carers' needs may not be adequately addressed.

In order to address the needs of carers of stroke survivors we developed a tailored intervention combining stroke education with strategies and techniques focussing on adjustment to stroke and caregiving. ${ }^{5}$

The aim was to determine whether it was feasible to recruit participants and to deliver and evaluate a biopsychosocial intervention for carers of stroke survivors as part of a randomized controlled trial (RCT).

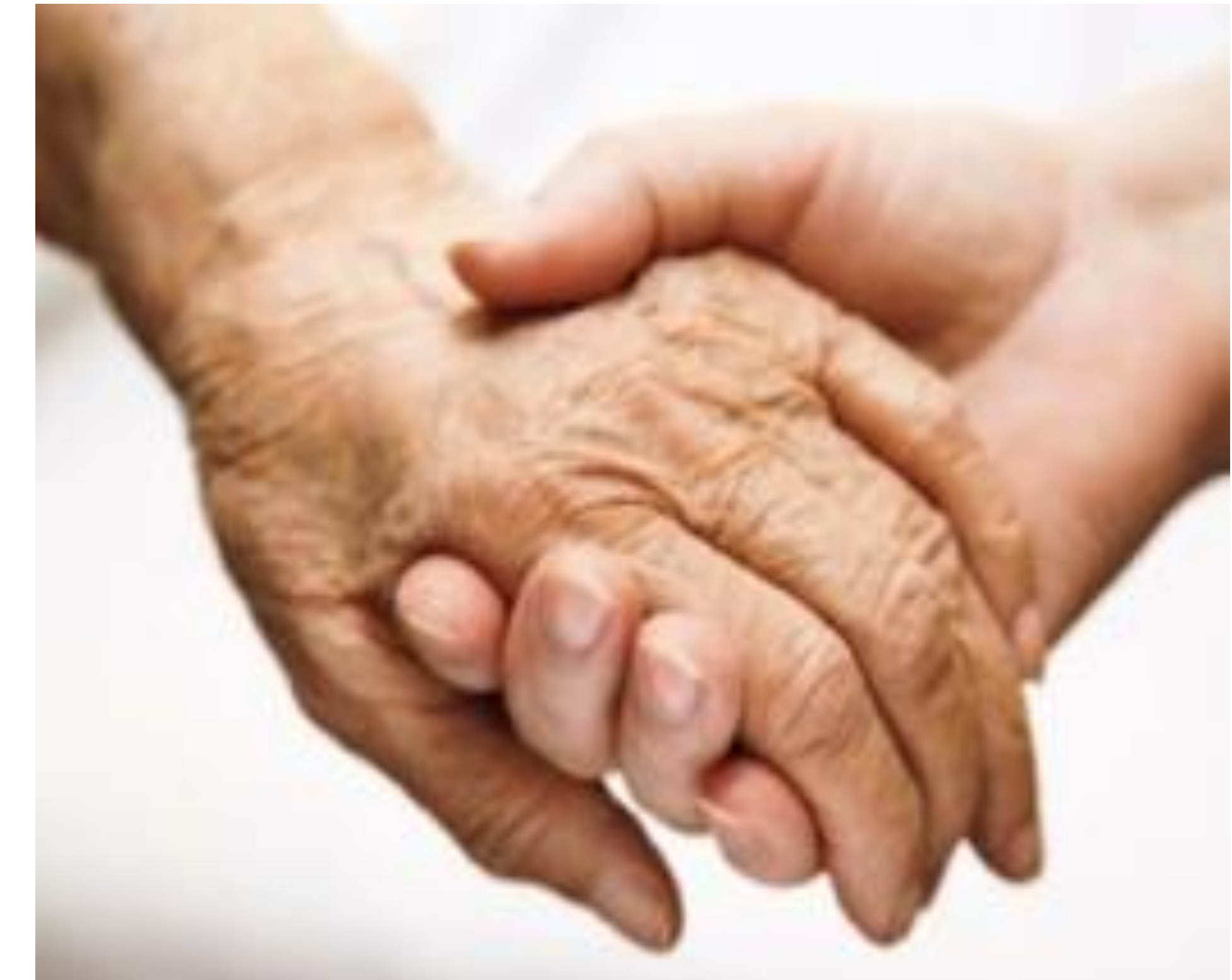

\section{METHODS}

\section{Feasibility RCT}

Patient/carer dyads within one year of stroke onset $(n=41)$ were recruited from the Stroke Service of Nottingham University Hospitals NHS Trust. Carers were randomised to either treatment (six-week intervention) $(n=18)$ or control group (usual care) ( $n=17)$. The intervention was delivered in two-hour sessions over six consecutive weeks, either in a group setting $(n=14)$ or on a 1:1 basis for those unable to attend groups $(n=4)$.

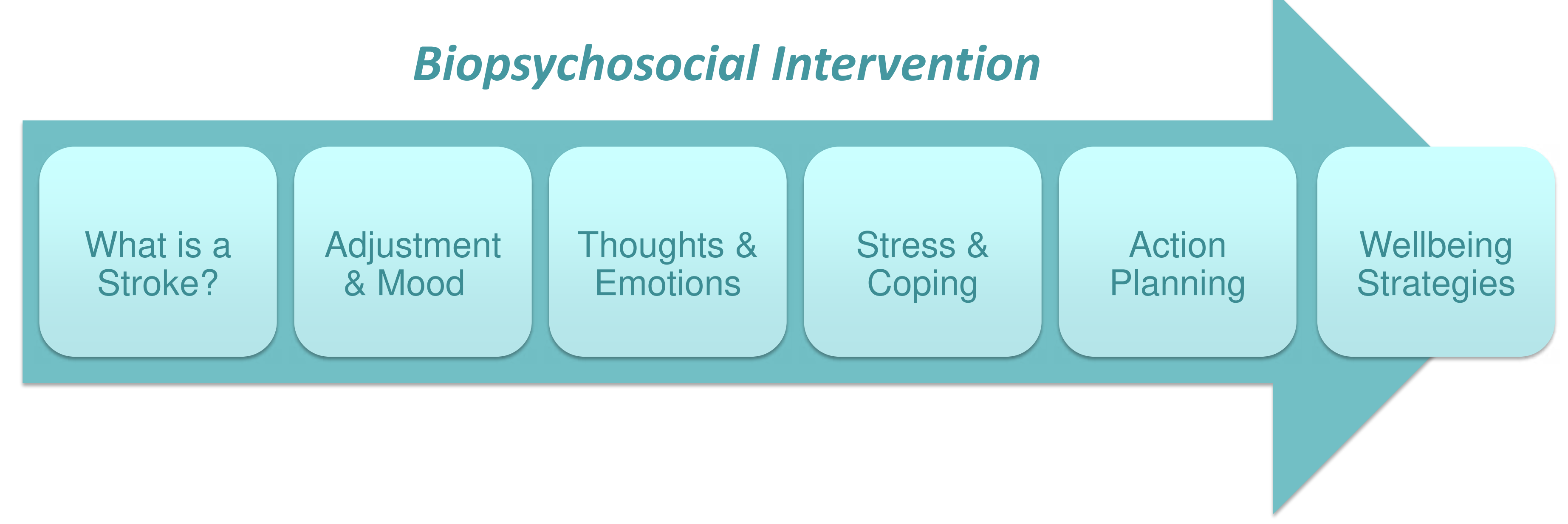

Intervention sessions consisted of educational presentations, group discussions, a workbook containing activities and strategies to practice between sessions, and relaxation exercises.

\section{Nested qualitative study}

Qualitative semi-structured interviews were conducted with purposively selected participants (based on age, gender and degree of stroke severity), in order to obtain feedback on all aspects of the study.

For the intervention group, interview questions explored views and experiences on intervention content, delivery mode, and perceived benefits. For the control group, questions explored the nature of usual care received and its impact on the carer.
Carer Outcome Measures (baseline $\&$ six months)

- Health-related quality of life (EuroQol EQ5D-5L) ${ }^{6}$

- Anxiety and depression (HADS) ${ }^{7}$

- Carer burden/strain (Caregiver Burden Scale (CBS)) ${ }^{8}$

\section{FLOW DIAGRAM}

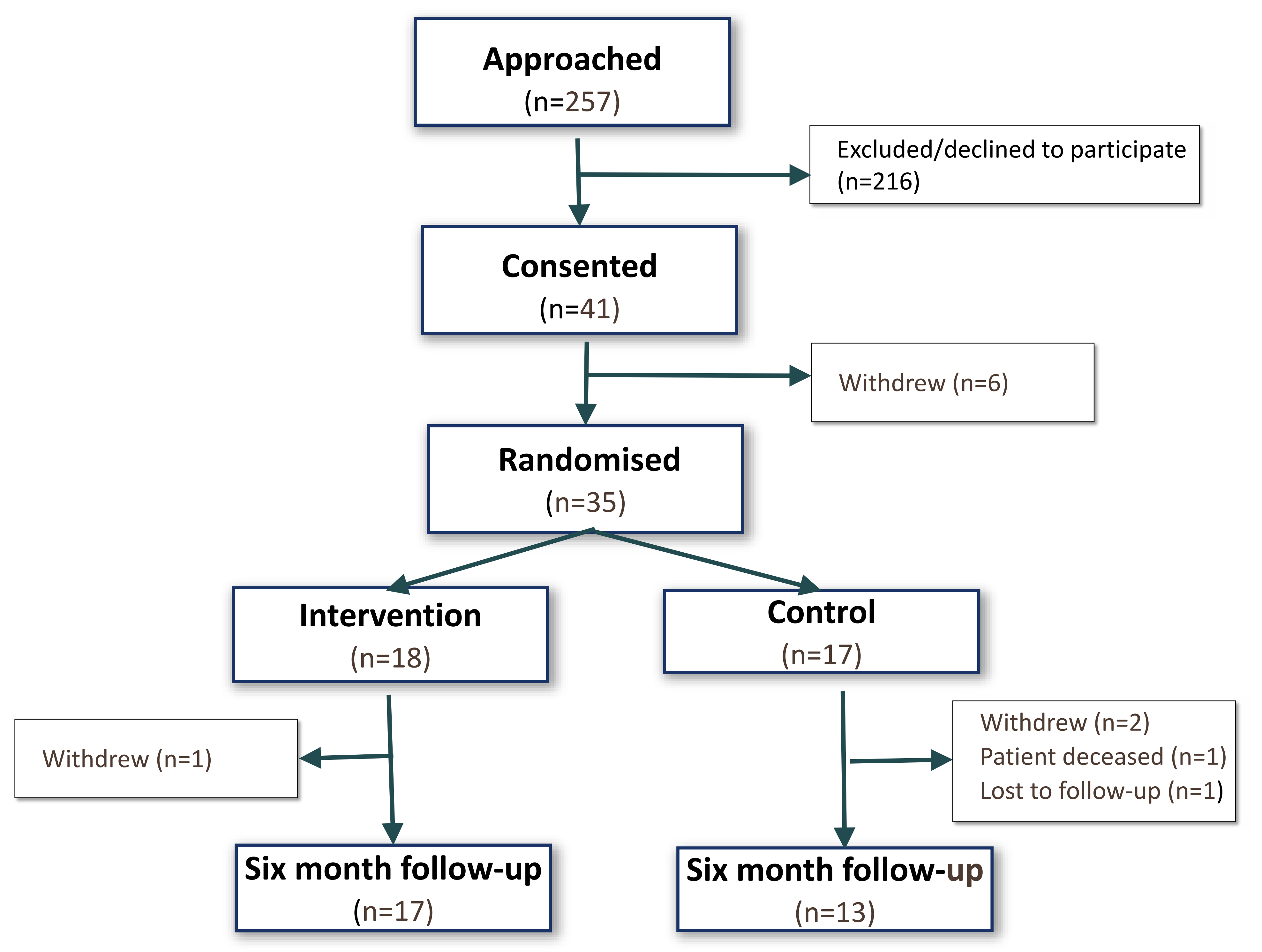

\section{RESULTS}

- Intervention group: Mean time from stroke to recruitment was 84 days. Carers were $56 \%$ female (mean age 63.3 years; range $30-82$ years).

- Control group: Mean time from stroke to recruitment was 77 days. Carers were 94\% female (mean age 61.9 years; range $39-80$ years).

- Mean number of intervention sessions attended across groups was 3.36 for group sessions, and 5.75 for $1: 1$ sessions.

- $86 \%$ of stroke carers were followed up at six months. $\star$ Improvements from baseline were shown on all outcome measures in both treatment and control groups.

$\star$ Feedback indicated that the intervention was acceptable to participants.

$\star$ It was shown to be feasible to deliver the intervention in both a group and a 1:1 format.

$\star$ Further exploratory and feasibility work is needed to identify the optimum timing for recruitment, format for intervention delivery and suitability of outcome measures. 